\title{
ANALYSIS AND RETRIEVAL OF PAINTINGS USING ARTISTIC COLOR CONCEPTS
}

\author{
Marchenko Yelizaveta, Chua Tat-Seng \\ National University of Singapore \\ E-mail: \{marchenk,chuats\}@,comp.nus.edu.sg
}

\author{
Aristarkhova Irina \\ National University of Singapore \\ E-mail: uspia@nus.edu.sg
}

\begin{abstract}
Traditionally artistic color concepts play an important role in the analysis of artworks, and provide valuable domain knowledge to guide the analysis and accurate retrieval of paintings. This paper presents an automated approach to analyzing and representing artistic color concepts such as color temperature, color palette and color contrasts in paintings domain. The color concept definitions rely on the widely accepted color theory of Itten. Our approach starts with the extraction of homogeneous color/texture regions from paintings, and employs image processing and machine learning techniques to characterize regions in terms of artistic color concepts. We evaluate the proposed method using collection of 1,000 paintings, which exhibit vast variety of painting styles and artistic color concepts. Experiments show that our method performs well for a vast variety of both spatial and non-spatial artistic color concept queries.
\end{abstract}

\section{INTRODUCTION}

Analysis of artistic color concepts constitutes a large body of expert knowledge in paintings domain. They also provide invaluable domain knowledge to guide the analysis and accurate retrieval of paintings based on artists, styles and periods etc, which are widely used for navigation through painting collections. Automatic analysis of artistic color concepts such as color temperature, color palette and color contrast also has the advantage that it facilitates automated annotation and retrieval of paintings by semantic color concepts in largescale artwork databases.

Recent systems for artwork retrieval [6,7] facilitate querying by color temperature and color contrast. The authors segmented images using K-mean clustering method and employed artistic color sphere to translate the average color of a region into a quantized space of artistic colors. Each of the artistic colors has the artistic color concepts assigned, which facilitates mapping of color onto the concept space.

Color averaging leads to the loss of information about the distribution of colors within a region. Such information is desirable for analysis of modern art paintings (post-impressionism, impressionism etc.) and paintings by a vast variety of artists (Van Gogh, Cezanne, Monet etc). In such paintings, contrasting colors and colors of different color temperature are placed close to each other at very fine level. Thus, the distribution of artistic color concepts, which pertain to each pixel within the color region, is non-uniform. Consequently, the use of averaged color to assign artistic color concepts does not model the artistic color concepts of a region accurately.

In the domain of western paintings, usage of color and their combination characterizes the artists and painting styles. The artists extensively used "light-dark" (or "chiaroscuro") contrast in Renaissance period, while the artists working in Post-impressionism and Fauvism paintings styles (Modern period) used complimentary contrast. Similarly, certain color expressions characterize the artists. Vincent van Gogh is known for his usage of complimentary colors and temperature contrasts. Berezhnoy et al. [4] performed automated analysis of complimentary colors in Van Gogh's paintings.

Hence, artistic color concepts serve as a cue for annotation of artworks with high-level categories of artist name, painting styles and fine art periods. In our previous work we introduced generic framework for annotation of paintings with artist names and painting style categories [11]. Within this framework, each painting is represented as a set of visual artistic concepts referring to artistic color, brush-work and composition concepts.

This paper builds on our previous framework by performing automated analysis to extract artistic concepts of color temperature, color palette and color contrast, and developing an automated system to retrieve paintings based on semantic color concepts. Section 2 gives definitions of artistic color concepts. Section 3 presents the methods for the artistic color concept analysis. Section 4 describes experiment results in paintings retrieval and Section 5 concludes the paper.

\section{COLOR CONCEPTS IN PAINTINGS}

We investigate artistic color concepts based on the theory formulated by Itten in 1960 [5]. Itten's color circle consists of three "primary" colors, three "complimentary" colors and six "tertiary" colors, giving rise to the color 
palette concepts. "Primary" palette represents the set of yellow, red and blue fundamental hues; "complimentary" palette represents the set of colors with green, orange and violet fundamental hues; and "tertiary" palette represents the other six fundamental hues of the color circle.

Fundamental hues of color circle vary through five levels of intensity and three levels of saturation. This gives rise to a total of 180 colors organized as a chromatic sphere. Fundamental colors are arranged along the equatorial circle of sphere, luminance varies along medians and saturation increases as the radius grows. Figure 1 shows the arrangement of colors in the chromatic sphere.

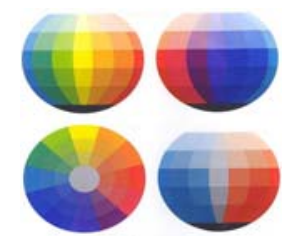

Figure 1. Itten's chromatic sphere

Color of the chromatic sphere with yellow-red-purple fundamental hues have "warm" color temperature, while colors based on green-blue-violet hues have "cold" color temperature. "Neutral" color temperature characterizes colors based on green-yellow and red-violet hues. "Neutral" colors become "cold" or "warm" depending on the surrounding colors. Properties of colors such as intensity and saturation influence the perceptual appearance of color temperature. To take this phenomenon into account, we introduce temperature strength weight that varies from 1 for fundamental hues to 0 for black, white and gray colors.

Color contrast is a relative measure defined for at least two colors. "Complimentary" contrast represents relationships between fundamental hues. In accord to Itten's theory, directly opposite hues of color circle have the strongest complimentary contrast. "Light-dark" color contrast accounts for the difference in color intensity of two colors. "Value" contrast reflects the relative difference of saturation property in colors pair. "Temperature" contrast reflects the interaction of different color temperatures.

In our proposed framework, we include the concepts of color temperature, color palette and color contrast at the artistic color concept level [11]. These artistic color concepts in turn helps to induce the next of the visual level give rise to the next abstract level concepts such as "warm", "cold", "expressive" and "chiaroscuro" at the painting level. "Warm" and "cold" abstract concepts characterize a painting in terms of the spatial properties of its "warm", "cold" color patches. "Expressive" abstract concept is usually assigned to paintings that exhibit strong "complimentary" and "temperature" contrast.

\section{ANALYSIS OF COLOR CONCEPTS}

We perform the automated analysis of artistic color concepts in three steps: image segmentation, analysis of the color concepts at the visual level that characterize image regions, and the analysis of the abstract artistic color concepts that apply to the whole image.

\subsection{Segmentation of paintings}

The analysis of color temperature and contrast concepts needs to take into account the spatial location of colors within a painting. Because of this requirement, global representations of color such as color histogram are inadequate for this type of analysis. To perform such localized analysis, image segmentation is necessary. We employ Blobworld segmentation [2] as it preserves color consistency of a region without producing a large number of fine regions due to variations of color and brush-work details.

\section{2 Artistic Color Concepts at Region Level}

\subsubsection{Low-level color and geometrical features}

To perform region analysis, we employ low-level color and geometrical features for each region. Geometrical features include normalized region area, coordinates of minimum bounding rectangle and region center, eccentricity, and solidity. As segmented regions may not be solid, we account for this by defining region solidity as the number of region pixels normalized by the number of pixels of the smallest convex hull of the region. In addition, we perform simple morphological operations by extracting contacting border between neighboring regions to store their location and normalized length. Currently each region maintains multiple dominant colors in our system. To calculate the vector of dominant colors, we employ CIE $\mathrm{L}^{*} \mathrm{u}^{*} \mathrm{v}$ color space [8]. The system calculates 316-bin color histogram and normalizes the color histogram by its maximum value. We select the top $\mathrm{k}$ values in the color histogram as the $\mathrm{k}$ dominant colors. We convert these colors to the corresponding reference colors in the Itten color space as follows:

$$
r e f=\arg _{M_{c}} \min _{1 \leq i \leq N} \operatorname{dist}(R c, M c(i)) \text { (1) }
$$

where dist denotes the normalized Euclidean distance, $R c$ denotes the CIE $\mathrm{L}^{*} \mathrm{u}^{*} \mathrm{v}^{*}$ values of dominant color, $M c(i)$ denotes the CIE $\mathrm{L}^{*} \mathrm{u}^{*} \mathrm{v}$ color values of color $i$ in the Itten's chromatic sphere, and $N$ denotes the number of Itten colors $(N=187$, including 5 shades of grey, black and white colors).

\subsubsection{Color temperature}


We employ a three-stage procedure to assign "warm", "cold" or "neutral" color temperature concept to a region. In the first stage, the system computes features to model the distribution of various color temperatures within a region. It averages the pixel values in $4 \times 4$ neighborhood in CIE L* $\mathrm{u}^{*} \mathrm{v}$ space, and finds its corresponding reference color in the chromatic sphere using Equation (1). Feature vector includes area and average temperature strength of pixels of each color temperature concept, color values of dominant colors in the HSI color space [8]. In this task we are concerned with distribution of color temperatures within a regions as color temperatures influence each other and their spatial distribution produces variety of percpetual effects.To handle this task, the system calculates the spatial coherence of image pixels of each temperature concept using a modification of the color coherence vector [3].

In stage 2, the system utilizes probabilistic SVM [9] and "winner takes all" strategy to assign color temperature concept for each region. Finally, the system refines the produced region labels. It selects regions with assigned "neutral" color temperature labels, calculates the length of the border with surrounding regions of "neutral", "cold" and "warm" color temperature, and updates the temperature label for region based on the longest common border.

\section{2. 3 Color contrast}

We perform color contrast analysis between two neighboring regions. As discussed above, our system represents color of a region as a set of $\mathrm{k}$ dominant colors. To effectively represent "complimentary", "temperature", "light-dark" and "value" color contrasts between two sets of dominant colors, we adopt the color-pair technique as discussed in [10]. This technique models two neighboring regions as a set of distinct color pairs based on the dominant colors from each region.

We performs the color contrast analysis in two steps. First, we select distinct color pairs. In our task, we treat a color pair as distinctive if it exhibits the strength of respective color contrast higher than a predefined threshold. To measure the strength of color contrast for a color pair, we find the two corresponding reference colors and study their relative location on the chromatic color sphere using 4 color contrast measures.

"Value" color contrast is defined as the normalized Euclidean distance between the absolute values of the reference color coordinates on the $\mathrm{X}$ plane of the chromatic color sphere. "Light-dark" and "complimentary" contrasts are defined similarly on the $\mathrm{Z}$ and Y planes of the sphere respectively. The definition of "temperature" contrast relies on the color temperature concept and the average temperature strength of two neighboring regions. Itten describes "warm-cold" pair as stronger temperature contrast as compared to "neutralcold" pair. We introduce this heuristics using:

$$
w(i, j)=\left\{\begin{array}{l}
1, \text { if } t_{i}={ }^{\prime} \text { warm }^{\prime} \& t_{j}=^{\prime} \text { 'cold' } \\
\left.0.5, \text { if }\left(t_{i}={ }^{\prime} \text { warm' } \| t_{i}=^{\prime} \text { cold' }\right) \& t_{j}=^{\prime} \text { 'neutal }\right) \\
0, \text { otherwise }
\end{array}\right\}
$$

The system calculates color temperature contrast as follows:

$$
\text { Ctemp }(i, j)=w(i, j) *(t s(i)+t s(j)) / 2
$$

Here $t s$ denotes the temperature strength of region $i$ and $j$ respectively.

In stage 2, we represent the whole region with respect to the four color contrast concepts. For each contrast concept, we combine into a normalized sum the area of selected color pairs weighted by the respective color contrast strength.

\subsection{Color palette}

Similarly to color temperature concepts, spatial distribution of colors, and their respective color palette concepts, within a region influences the overall perception of color palette of a region. To analyze the "primary", "complimentary" and "tertiary"color palette concepts, the system calculates the color coherence vector of a region based on 316-bin color histogram in CIE $\mathrm{L}^{*} \mathrm{u}^{*} \mathrm{v}$ color space. For each color bin, the system finds its fundamental hue using Equation (1) and extracts color palette category based on the chromatic sphere. Next, for each color palette concept, the system calculates the weighted sum of the coherent and non-coherent pixels in the respective color bins and normalizes them over the number of region pixels.

\section{3 Abstract Color Concepts at Image Level}

Artistic color concepts of the regions serve as the basis for the analysis of "warm", "cold", "expressive" and "chiaroscuro" abstract color concepts assigned to the whole image. To capture the color concepts of the abstract level, we extract the values of the respective color concepts for the image regions and combine them into the normalized sum of the color concept values weighted by the geometrical region properties.

\section{EXPERIMENTS}

In our experiments we use 1,000 paintings of modern art in five paintings styles from [1]. The image size in the collection is $384 \times 256$. We evaluate the proposed method in paintings retrieval by artistic color concepts. As a baseline, we employ simple segmentation technique that divides an image into nine equal parts. We refer to the variations of the proposed method based on the baseline and Blobworld segmentation techniques as Test1 and 
Test2 respectively. To measure the accuracy of labeling with color temperature concepts we use a ground truth of 4,500 regions and found the accuracy of $93 \%$ and $86 \%$ for Test 1 and Test 2 respectively.

Retrieval system combines artistic color concepts and geometrical features of the regions to index paintings. For color temperature and color palette queries, the system takes into account the region area, location, solidity and eccentricity. For queries with artistic color contrast concepts, the system considers the area and solidity of regions, location and length of the contacting border.

Table 2 contains examples of four query groups. Each group contains 3 to 5 queries.

Table 2. Examples of queries

\begin{tabular}{|c|l|c|}
\hline No & \multicolumn{1}{|c|}{ Query } & Group \\
\hline 1 & Painting with cold region at the top & 3 \\
2 & Complimentary contrast on the left & 4 \\
3 & Temperature contrast in the center & 3 \\
4 & Light-dark contrast at the top & 4 \\
5 & Color of primary palette in the center & 3 \\
6 & Painting in warm colors. & 1 \\
7 & Painting with chiaroscuro technique. & 2 \\
8 & Expressive painting. & 2 \\
9 & Painting in cold Colors of primary & 1 \\
& palette & \\
\hline
\end{tabular}

Group 1 covers queries with abstract concepts of color temperature and color palette. Group 2 represents queries with abstract color contrast concepts, while Group 3 incorporates spatial queries of the color temperature and color palette concepts. Lastly, Group 4 represents spatial queries of the color contrast concepts. On average, the expert-provided ground truth for the test queries contains 140 images.

Table 3 shows the system performance based on Mean Average Precision (MAP) metrics that allows comparison of queries with variable ground truth size.

Table 3. Evaluation of the system performance

\begin{tabular}{|c|c|c|c|c|}
\hline & Group1 & Group2 & Group3 & Group4 \\
\hline Test 1 & 0.768 & 0.399 & 0.601 & 0.313 \\
\hline Test 2 & 0.798 & 0.732 & 0.693 & 0.685 \\
\hline
\end{tabular}

Test1 and Test2 do not differ significantly for Group1 queries as this query group does not require spatial information about image regions. The difference in the relative performance of Test 1 and Test 2 is most apparent in Group2 and Group4. These query groups require appropriate information about spatial color distribution. Overall, Test 2 performs better due to more consistent neighborhood information provided by Blobworld segmentation method. The MAP of Test 2 across all queries is 0.73 . Figure 2 shows the top six images retrieved by the query "Painting in warm colors".

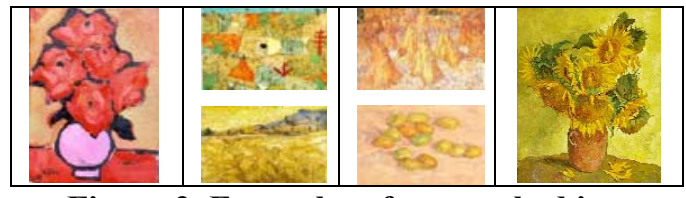

Figure 2. Examples of top-ranked images

\section{CONCLUSIONS}

In this paper, we propose an automated approach that utilizes domain knowledge of arts domain to analyze and retrieve paintings. In particular, it uses major artistic color concepts in arts domain to represent semantic categories for paintings retrieval and provide important cues for automatic classification by artist name and painting style. The method utilizes spatial information about colors that accounts for a variety of painting styles. The method has been found to be effective. In our future work, we will employ artistic color concepts for the purpose of such classification.

\section{REFERENCES}

[1] Artchive, http://www.artchive.com/ftp_site.htm

[2] C. Carson, S. Belongie, "Blobworld. Image segmentation using expectation maximization", IEEE PAMI, 24(8): pp. 1026--1038, 2002.

[3] G. Pass, R. Zabih, J. Miller. Comparing Images Using Color Coherence Vectors. ACM MM, pp. 65-73, 1996.

[4] J. Itten, "The Art of Color", Reinhold Corp., 1961.

[5] J. M. Corridoni, A. Del Bimbo, and P. Pala, "Retrieval of Paintings Using Effects Induced by Color Features", CAIVD'98, pp. 2-11,1998.

[6] J. Lay, L. Guan, "Retrieval of color artistry by color concepts," IEEE Trans. on IP, vol. 13(2), pp. 326-339.

[7] R. C. Carter, "CIELUV Color Difference Equations for Self-Luminous Displays", Color Research and Applications (8), pp. 252-253.

[8] Chakrabartty, S. and Cauwenberghs, G. "Forward Decoding Kernel Machines,” IEEE PR, 2002.

[9] T.-S. Chua, S.-K. Lim. "Content-based retrieval of segmented images”. ACM MM, pp.211-218,1994.

[10] Y. Marchenko, T.-S. Chua, "Retrieval and Representation of Paintings Based on Art Concepts", ICME 2004.. 